\title{
Review of: "Placental DNA methylation signatures of maternal smoking during pregnancy and potential impacts on fetal growth"
}

\author{
Esha Bhattacharjee ${ }^{1}$, Jagyashila Das ${ }^{1}$, Piyali Mondal $^{1}$, Arindam Maitra $^{1}$ \\ 1 National Institute of Biomedical Genomics (NIBMG)
}

Potential competing interests: The author(s) declared that no potential competing interests exist.

In this study, the authors have reported an association between fetal-side placental DNA methylation (DNAm) changes and maternal smoking during pregnancy (MSDP), using a large sample size spanning across seven independent studies on different ethnic groups. They performed an inverse variance fixed effects meta analysis. Overall, they found $443 \mathrm{CpG}$ sites to be associated with any or sustained MSDP. The CpGs were mapped to 284 genes, which were enriched for cell adhesion molecules, asthma, BMI, blood pressure, antipsychotic agents as inferred from dbGAP. Using these 443 MSDP-associated CpGs, they performed eQTM analysis and mapped $40 \mathrm{CpGs}$ with the strongest association with expression of nearby mRNA. They found 9 biological pathways significantly enriched among the eQTM mapped genes, which were primarily involved in inflammatory activity.

They also identified 61 SNPs associated with birth outcomes within $0.5 \mathrm{Mb}$ of $51 \mathrm{CpGs}$ suggesting involvement of MSDP in fetal growth regulation. They also found $121 \mathrm{CpGs}$ to be associated with gestational age at birth. Twenty-five CpGs were found to be associated with birth weight, $11 \mathrm{CpGs}$ with birth length and 2 CpGs with head circumference.

Finally, the investigators assessed whether the DNA methylation signatures of MSDP found in placenta were tissue-specific or also present in cord blood by evaluating the presence of these signatures in data from a published cord blood study of PACE Consortium. They identified 4 CpGs with significant association in both the placental tissue and cord blood. However, 2 of them showed opposite direction of effect i.e. in one tissue the CpG was hypermethylated while in the other it was hypomethylated. This suggests MSDP also results in specific tissue-specific epigenomic changes in the DNA in addition to consistent changes between these two tissues.

The study is an important one with significant strengths. The sample size is large, drawn from several independent studies on different ethnic groups. The results are backed by elaborate secondary analyses involving mRNA expression, functional and phenotype enrichment and analysis of overlap with GWAS hits for reproductive outcomes. Overall, DNAm signatures of MSDP in placenta, identified by the investigators, show promising mechanistic relevance, which needs to be validated in future studies. Some of the limitations of the study worth mentioning are the lack of inclusion of populations of diverse ancestries and 
the self-reported status of MSDP used for analysis.

In addition to the above, there are a few concerns about the report.

In the gene-set enrichment analyses, the authors reported that enrichment analysis with the 284 gene-set identified a list of genes whose methylation level were altered across pregnancy due to MSDP. Since, the study was cross-sectional and not temporal, so concluding remarks of the authors for the enrichment analysis with the first gene-set is not clear.

In the association study between DNA methylation and birth outcomes, the authors conducted inversevariance weighted fixed effect meta-analyses. From the forest plot, it's clearly visible that not only the weight of the study (i.e. sample size) varied among the cohorts but there was also heterogeneity at some of the significantly associated markers. Thus, before making a generalized comment for such loci (eg. association of cg27402634 with larger birth weight; I²: 55.17\%), those results should be cross-validated by random-effect meta-analysis.

Apart from these, there were a few lack of clarity in the report viz. the numbers of autosomal SNPs evaluated for the identification of MSDP-associated CpGs in placenta located within 1Mb window of previously reported birth outcome-associated SNPs; whether hypomethylation at cg27402634 and cg20340720 were associated with larger or shorter birth weight (BW) and whether hypomethylation at cg26843110 was associated with longer or shorter gestational age (GA). Moreover, the report of hypomethylation at cg26843110 resulted in decreased expression of CSK is difficult to comprehend if it is a promoter CpG for CSK gene. 